\title{
Comparison of Two Novel Techniques for Iris Centering in Ocular Prosthesis
}

\author{
Dhananjay Arora ${ }^{1}$ Shefali Singla ${ }^{1} \quad$ Lalit Kumar $^{1} \quad$ Komal Sehgal $^{1} \quad$ Puneet Sharma ${ }^{1}$ \\ 1Department of Prosthodontics, Dr. Harvansh Singh Judge Institute \\ of Dental Sciences, Panjab University, Chandigarh, India

\begin{abstract}
Address for correspondence Dhananjay Arora, BDS, Room No 126 , Department of Prosthodontics, Dr. Harvansh Singh Judge Institute of Dental Sciences, South Campus, Panjab University, Sector 25, Chandigarh 160014, India (e-mail: dhananjay29may@yahoo.com).
\end{abstract}

\begin{abstract}
Keywords

- customized iris

- iris centering

- ocular prosthesis

Eyes are among the first features of the face to be noticed. Loss of an eye due to congenital, traumatic, or pathologic etiologies causes disfigurement and loss of sensory feedback. It leaves a psychological impact on the patient; thus, prosthesis should be provided at the earliest to raise the spirits of the afflicted. Iris positioning is one of the important steps in fabricating customized ocular prosthesis. In facial asymmetry cases, comparison of both irises together can be a major disadvantage. This case report illustrates the use of a regular eyeglass in one technique and the use of a bioart facebow in the second technique for centering of iris in a customized ocular prosthesis to overcome the disadvantages of earlier used standard techniques (McArthur's ocular locator and fixed calliper, pupillometer, visual judgement, and graph grid) of iris centering.
\end{abstract}

\section{Introduction}

Defects of the eye may include either en bloc removal of the entire orbit, that is, exenteration or removal of scleral defects, that is, enucleation of only the eyeball. ${ }^{1}$ In the Indian subcontinent, trauma, tumors and congenital absence of orbit are the main causes of such defects. Besides suffering a loss of vision, these patients become esthetically and psychologically handicapped and are not well-accepted in society. In defects that are not amenable to surgical correction, a prosthetic eye can prove beneficial. This may involve replacing the entire eye or simply an indwelling eye that replaces the outer scleral portion.

Indwelling eyes are made to precisely fit the confines of the ocular socket of the patient. They mainly comprise of the sclera and iris and are colored and polished to make the prosthesis look natural. They not only provide esthetics, but also protect the eye cavity, thereby preventing infections.

Prosthetic eyes can be prefabricated or custom made, with the latter offering better fit and esthetics. A stock ocular prosthesis can be provided as an interim prosthesis or when time limitation exists, and the cost is an important factor. Stock ocular prosthesis has many disadvantages, such as poor fit, improper shade matching, iris positioning, and so on, whereas a custom-made ocular prosthesis increases the adaptiveness, movement of the eye ball, and exactly matches the iris position as that of the adjacent natural eye.

Prosthetic replacement of lost eye presents with many challenges, with the most important being accurate positioning of iris. Any asymmetry in positioning causes a squinted eye appearance which leads to unaesthetic results. Literature reports various methods like pupillometer, ${ }^{2}$ caliper measurement, ${ }^{3}$ graph grid, ${ }^{4}$ ocular locator and calipers, ${ }^{5}$ dividers, ${ }^{6}$ anatomic tracing ${ }^{7}$ and visual judgement ${ }^{8}$ for location of iris position in ocular positioning. Visual judgement is subjective, and stability of other documented devices is doubtful, especially in patients with facial asymmetry.

The objective of the proposed techniques is to achieve predictable positioning of the iris in order to enhance the final esthetic effect of the prosthetic eye and achieve a conversational gaze that can form a reliable source of nonverbal expression on the patient's face.

\section{Case Report}

A 70-year-old male patient reported to the Department of Prosthodontics and Crown and Bridges with a chief complaint of missing teeth as well as an ocular defect.
DOI https://doi.org/ $10.1055 / \mathrm{s}-0040-1712061$ ISSN 2321-1482.
License terms

()(1) $\Theta \circledast$ 
The patient had lost his eyesight in an accident and in spite of multiple surgeries his eyesight could not be restored. On examination, the sclera was present, but the iris was discolored. The muscle function of both the upper and lower eyelid seemed normal. The patient was counselled about the benefits of prosthetic eye and his consent was taken for the procedure.

\section{Procedure}

- The patient was seated in an erect position to allow the tissues involved in the defect to be recorded in their natural drape.

- A stock conformer was used to make initial impression using alginate (Zelgan, Dentsply Inc.).

- Final impression was recorded using light body (Express VT Light Body, 3M ESPE) and custom tray.

- The impression was invested in alginate to obtain an index. A wax conformer was fabricated by flowing molten wax in the index, tried in the patient's eye socket, and adjusted for desired volume, retention and comfort.

\section{Centering the Iris on Wax Pattern}

Two techniques have been described to determine and duplicate the exact dimensions and location of iris in ocular prosthesis.

\section{Technique 1: Using a Customized Eyewear}

- In this technique, a plain pair of eyeglasses was customized using plastic scales.

- Two pairs of scales (Camlin, Pidilite Industries Ltd, Mumbai, India) were cut up to $6 \mathrm{~cm}$ and $4 \mathrm{~cm}$ length for iris location.

- $6 \mathrm{~cm}$ scales were fixed to the top of eyewear in horizontal direction, starting from the central connector of the two sides of frame.

- To standardize the starting point, a diamond bur was placed at both ends of the connector and scales were attached on the bur, indicating start point.

- Two scales of $4 \mathrm{~cm}$ length were placed vertically to determine the vertical placement of iris.

- Midline of face was marked, and the patient was asked to gaze toward a fixed point placed parallel to his eyes and kept at a distance.

- The iris of the normal eye was marked on the forehead by keeping a scale on the medial and lateral ends of iris and this distance was measured using Boley's Gauge (-Fig. 1).

- The distance between medial and lateral end of iris was also measured.

- These two markings were transferred onto the defect side with the help of Boley's Gauge.

- The eyewear was put on the patient and the marking were transferred onto the eyewear (-Fig. 2).

- The vertical dimension of iris was determined with vertically placed scales on the nondefect side and markings were then transferred to defect side [ - Fig. 2].
- By getting a box by intersection of the vertical and horizontal lines, the iris button was placed onto the wax pattern and centered within the box made by grids (-Fig. 3).

- The limitations of this method are that facial asymmetry including any nasal or auricular anomaly or defect will interfere with symmetrical position of eyeglasses on defect and nondefect sides.

\section{Technique 2: Using a Bioart Facebow with a Nasion} Relator

- In this method a facebow (Bioart Standard Facebow, Bioart Industry, Sao Paulo, Brazil) with a nasion as the third point of reference was used.

- The nasion marker was removed and a $15 \mathrm{~cm}$ scale was fixed to the nasion rod, so that $7.5 \mathrm{~cm}$ marking coincided with the center of the rod (-Fig. 4).

- Midline of face was marked.

- The patient was asked to gaze toward a fixed point placed parallel to his eyes and kept at a distance.

- The same technique for measurement of iris on the defect side, as in the previous one, was followed.

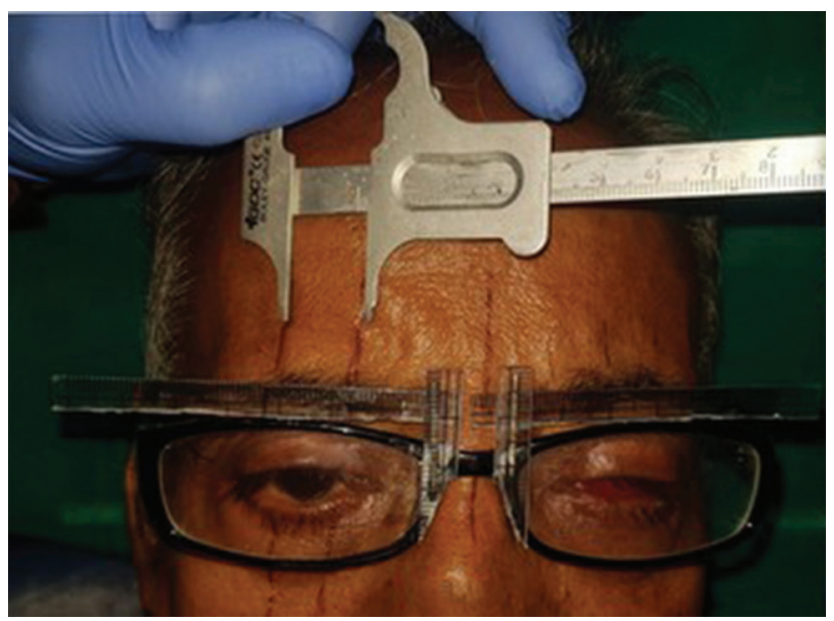

Fig. 1 Midline and iris position marked on forehead.

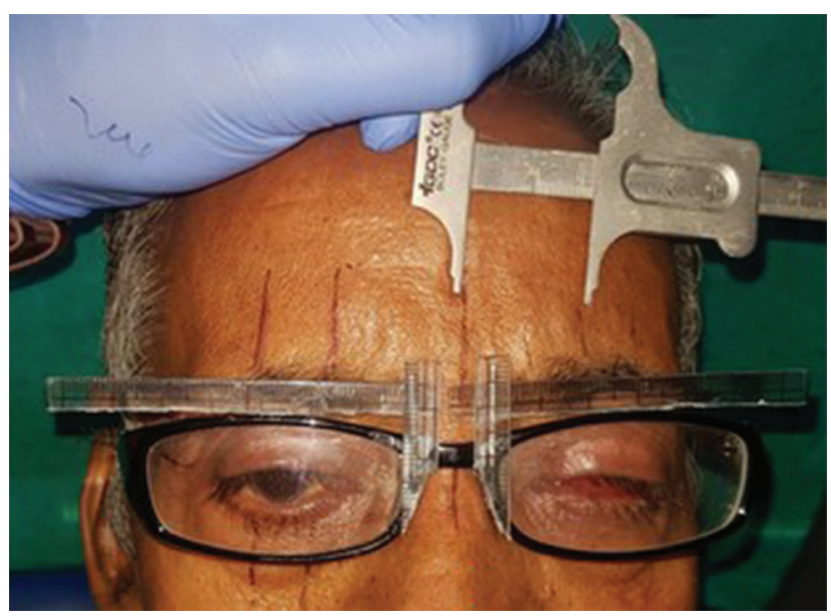

Fig. 2 Transferring the marking on eyewear. 


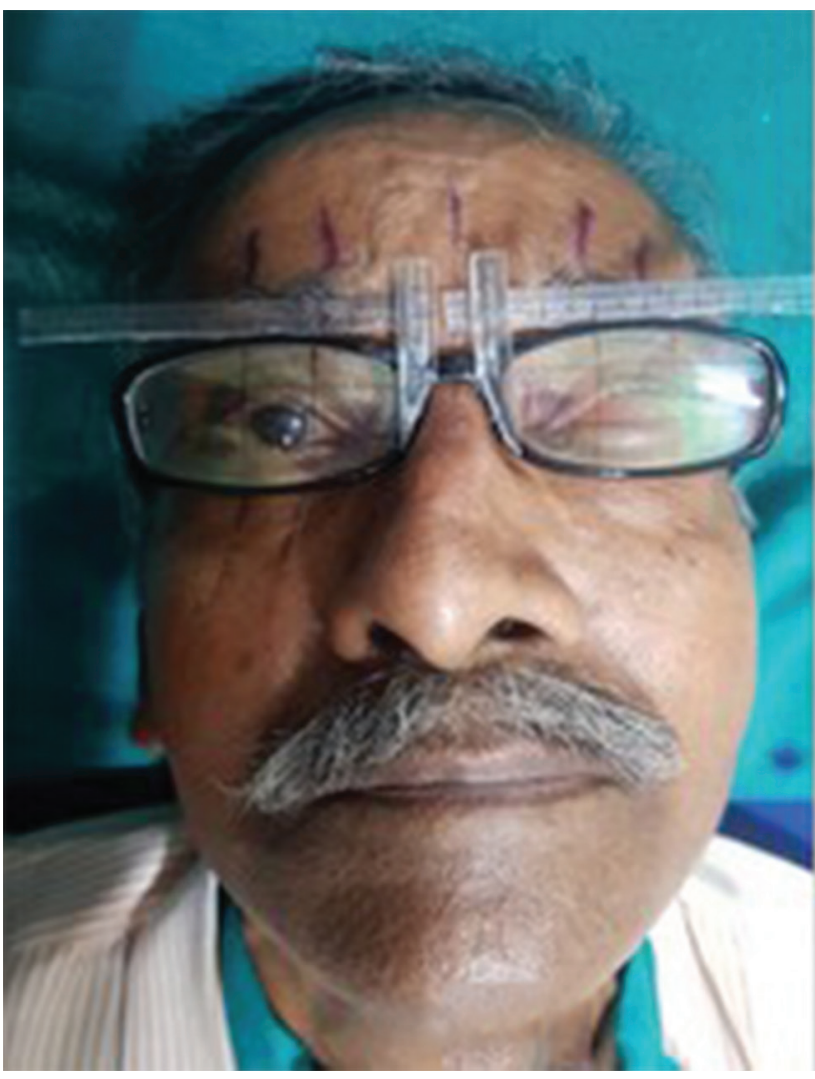

Fig. 3 Iris button position according to the grid formed on eyewear.

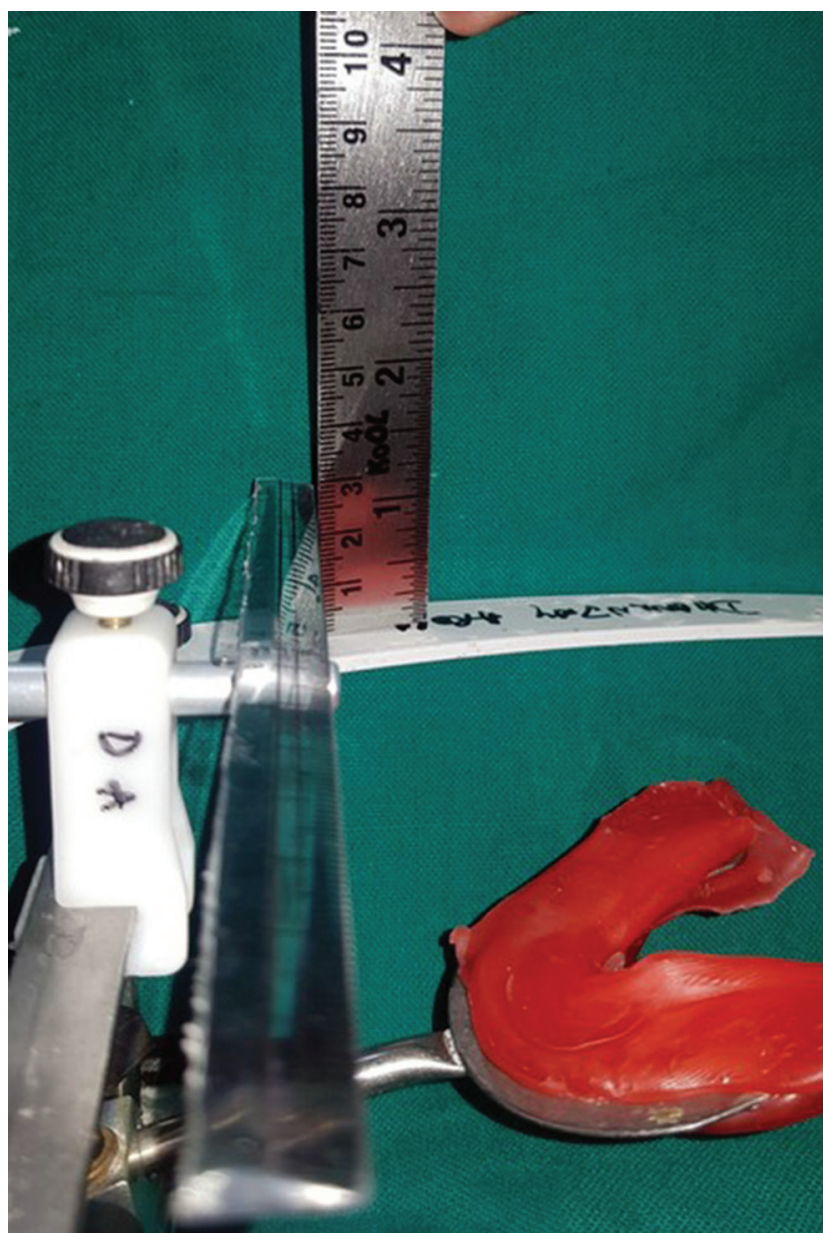

Fig. 4 Facebow with scale mounted on nasion.
- Now, instead of eyewear, the facebow was placed on the patient, with the bitefork attached to maxillary teeth with aluwax. The bite fork acts as the stabilizing third point, while the two earpieces of the facebow provide two posterior stabilizing points.

- The scale mounted on the nasion was adjusted at the level of lower eyelid.

- The $7.5 \mathrm{~cm}$ marking on the scale coincides with the midline of face.

- The lines on the face locating iris position on defect side were transferred onto the scale and the markings noted.

- The patient was placed in supine position and asked to gaze at infinity.

- The iris locator was placed according to the markings on the scale on the defect side (-Fig. 5).

\section{Comparison of the Result of Centering Using Two Techniques}

The outcome of the two methods of centering the iris was compared using patient's photograph and standardized by clicking from the same distance. The camera was mounted on a tripod according to the center of the dental chair. ISO values, shutter speed, and aperture were standardized

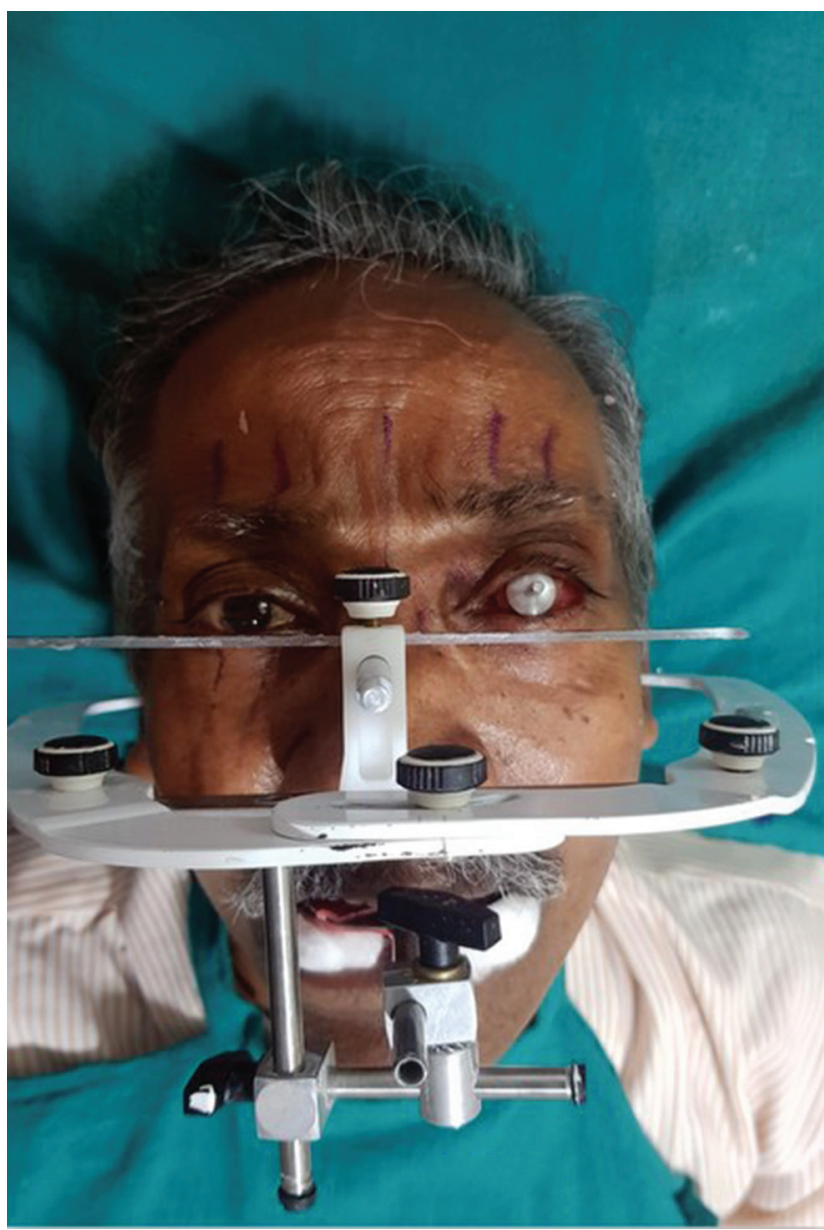

Fig. 5 Iris location. 
according to the light availability, and the zoom was also set according to the patient's position.

The patient was asked to gaze at a point placed at the level of his eye at some distance. First, the photograph without the prosthesis (-Fig. 6A) was taken and thereafter with prosthesis, with iris positioned using both the techniques (-Fig. 6B, C). The photographs of patient with prosthesis were superimposed with the photograph without prosthesis on ADOBE PHOTOSHOP SNAPSEED SOTWARE.

The outlines of the face were matched in the photographs superimposed on each other. Then, the photograph superimposed with prosthesis was lowered at the level of lower eyelid, so that comparison could be made out.

\section{Result}

The superimposed photograph was showcased onto a projector screen in the presence of 15 clinicians at the Department of Prosthodontics, Crown and Bridges. The photograph with prosthesis using eyewear was marked as 7a and the photograph with prosthesis using facebow was marked as 7a. As many as 10 clinicians found -Fig. 7B to be more accurate, three were not sure, and two found - Fig. 7A more accurate.

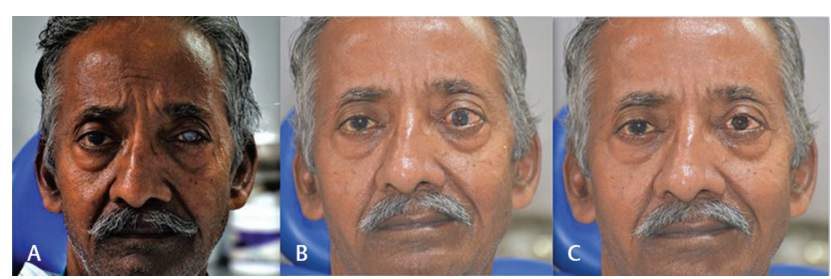

Fig. 6 (A) Without prosthesis, (B) iris located with eyewear, and (C) iris located with facebow.

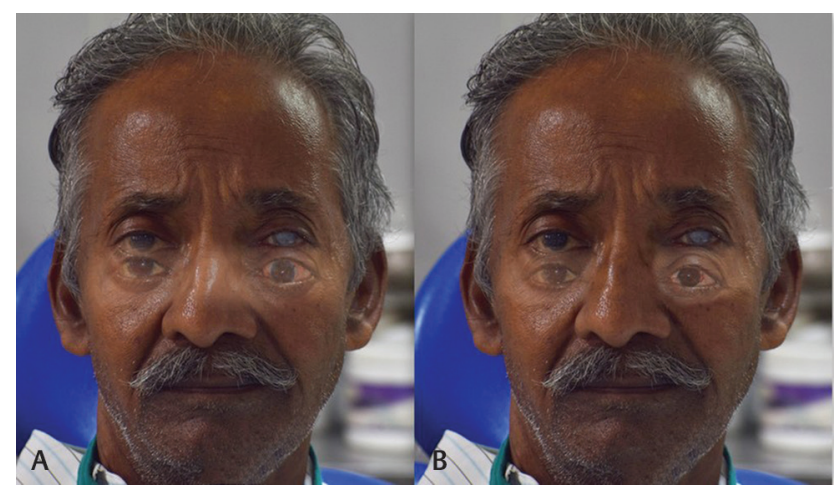

Fig. 7 Superimposed pre and postphotograph of iris located $(\mathbf{A})$ with eyewear and (B) with facebow.
The patient and his wife were also satisfied with 7b. Based on the subjective judgement of the clinicians, it was unanimously made out that the prosthesis fabricated using a facebow centered the iris accurately. The iris located with an eyewear was somewhat medially placed. Both the prostheses were esthetic, but the prosthesis made with iris located using a facebow was more accurate.

\section{Discussion}

Different methods have been suggested to determine the size and position of the iris. Benson advocated a method of determining the size and position of the iris by visual judgment. Since iris positioning is a technique-sensitive procedure, visual assessment alone may not be reliable and may incorporate parallax errors. Roberts suggested the use of a pupillometer for exact alignment of the pupil in the ocular prosthesis. ${ }^{2}$ Pupillometer is an instrument consisting of two parallel cylinders with positive lens for locating the pupil. However, the technical difficulty of making a pupillometer available in every clinical setup is a limitation for using this method. McArthur used an ocular locator and fixed calliper for the accurate placement of the prosthetic eye in an orbital prosthesis. ${ }^{5}$ Visual judgement is subjective, and stability of the documented devices is doubtful, especially in patients with facial asymmetry. ${ }^{8}$ Shetty $\mathrm{P}$ used an inverted springbow with orbital pointer at ala and fork mounted, with measuring scale to determine vertical and horizontal dimension and location of iris. ${ }^{9}$ The stabilization of device in this method depended on earpieces only and was liable to displacement in supero-inferior dimension. Also, there wasn't any reference positioning for recording vertical dimension of iris.

Two techniques have been proposed to overcome these limitations. Eyewear method is simple and easy to execute, but it relies on facial symmetry in terms of nose and ear for its correct positioning. In prosthesis fabricated using an eyewear, iris was placed a bit medially, which can be due to discrepancies in transferring the marking from face onto the eyewear. This is subjective and may vary from one clinician to another. Facebow stabilized anteriorly with bite fork attached to maxillary teeth is a more reliable and repeatable modification. Since two posterior (earpieces) and one anterior (bite fork) reference points are used to stabilize the frame on face, chances of error or movement while locating iris positioning are minimized. Instead of scale, a grid can also be attached to the nasion rod for better $2 \mathrm{D}$ accuracy. 


\section{Conclusion}

The technique described here has attempted at positioning the iris, virtually eliminating the subjective errors arising out of visual illusion by using different methods. The patient satisfaction and acceptance were further improved using manual paint on technique for characterization of iris and sclera.

\section{Conflict of Interest}

None declared.

\section{Reference}

1 Raflo GT. Enucleation and evisceration. In: Tasman W, Jaeger E, eds. Duane's Clinical Ophthalmology, vol 5, (ed 2). Philadelphia, PA: Lippincott; 1995:1-25

2 Roberts AC. An instrument to achieve pupil alignment in eye prosthesis. J Prosthet Dent 1969; 22(4):487-489
3 Brown KE. Fabrication of orbital prosthesis. J Prosthet Dent 1969; 22(5):592-607

4 Guttal SS, Patil NP, Vernekar N, Porwal A. A simple method of positioning the iris disk on a custom-made ocular prosthesis. A clinical report. J Prosthodont 2008; 17(3):223-227

5 McArthur DR. Aids for positioning prosthetic eyes in orbital prostheses. J Prosthet Dent 1977; 37(3):320-326

6 Jooste $\mathrm{CH}$. A method for orienting the ocular portion of an orbital prosthesis. J Prosthet Dent 1984; 51(3):380-382

7 Raflo GT. Enucleation and evisceration. In: Tasman W, Jaeger E, eds. Duane's Clinical Ophthalmology, vol 5, (ed 2). Philadelphia, PA: Lippincott 1995:1-25

8 Benson P. The fitting and fabrication of a custom resin artificial eye. J Prosthet Dent 1977; 38(5):532-538

9 Shetty PP, Chowdhary R, Yadav RK, Gangaiah M. An iris positioning device and centering approach: a technique. J Prosthet Dent 2018; 119(1):175-177 